\title{
Classifications in Brief: Rüedi-Allgöwer Classification of Tibial Plafond Fractures
}

\author{
T. David Luo MD, J. Matthew Eady PharmD, Arun Aneja MD, PhD, \\ Anna N. Miller MD
}

Received: 8 August 2016/Accepted: 19 December 2016/Published online: 4 January 2017

(C) The Association of Bone and Joint Surgeons ( 2017

\section{History}

According to Michelson et al. [17], the French word for pestle, "pilon," was first used by Étienne Destot in 1911 as a metaphor for the mechanical function of the distal tibia on the talus. Subsequent descriptions of fractures of the tibial plafond adopted the term "pilon" to describe the explosive nature and axial compression mechanism of these injuries, in which the tibia acts as a pestle and is driven vertically into the talus [10, 21, 22]. Fractures of the tibial plafond commonly result from high-energy trauma with axial load, sometimes leading to severe bone loss and soft tissue injury [3, 5, 15]. They are relatively rare, accounting for $1 \%$ to $10 \%$ of lower leg or tibial fractures, but are substantial injuries that can result in persistent

Each author certifies that he or she, or a member of his or her immediate family, has no funding or commercial associations (eg, consultancies, stock ownership, equity interest, patent/licensing arrangements, etc) that might pose a conflict of interest in connection with the submitted article.

All ICMJE Conflict of Interest Forms for authors and Clinical Orthopaedics and Related Research ${ }^{\circledR}$ editors and board members are on file with the publication and can be viewed on request.

T. D. Luo ( $\square)$, J. M. Eady

Department of Orthopaedic Surgery, Wake Forest Baptist

Medical Center, Winston-Salem, NC 27157, USA

e-mail: tluo@wakehealth.edu

\section{A. Aneja}

Department of Orthopaedic Surgery, University of Kentucky, Lexington, KY, USA

A. N. Miller

Department of Orthopaedic Surgery, Washington University

School of Medicine, St. Louis, MO, USA disability if not fixed properly. Many of these fractures leave the patient with persistent pain, limitations in ROM, and residual disability, especially when the fracture patterns involve severe articular comminution [18, 26, 30]. Complications after surgical treatment and inconsistency in achieving fracture union during the early half of the last century led many physicians to consider these fractures as "not amenable to surgery" [22]. As a result, patients had to live with the persistent disability.

One of the first pivotal shifts in the approach to treating pilon fractures came from the proposed techniques and results published by Rüedi and Allgöwer in 1968 [23]. They noted from previous studies that nonoperative treatment was likely to result in secondary joint displacement and worse outcomes. Rüedi and Allgöwer postulated that by adhering to strict aseptic technique and applying four operative stages, internal fixation of pilon fractures would result in the best-possible anatomic reconstruction. This staged principle was applied in a series of 84 comminuted pilon fractures in 82 patients [21]. Rüedi and Allgöwer's first step calls for restoration of the length of the fibula to assist in fracture reduction of the tibia. They noted that $60 \%$ of all cases involved a concomitant transverse or oblique fracture of the fibula. An initial approach to the tibia was possible in the remaining $40 \%$ of cases. The second step involves anatomic restoration of the distal articular surface of the tibia. They commented on the "jigsaw puzzle" nature of severely comminuted fractures in half of all patients and recommended using the talus as a guide to reconstruct the tibia. Next, autologous bone graft can be used to fill the metaphyseal bone defect and support the articular surface to prevent collapse. Finally, buttress plate fixation can be applied to the medial side of the tibia to prevent late-onset varus deformity. 
Although there are numerous modern techniques for pilon fixation, the concepts presented by Rüedi and Allgöwer still form the foundation of the surgical approach to these injuries $[24,30]$. Furthermore, their contributions to the management of pilon fractures included not only surgical principles, but also the introduction of a classification system still widely used [26, 27].

\section{Purpose}

Rüedi and Allgöwer reported the results from their original cohort with up to 9 years of followup and categorized the fracture patterns that would become the classification schema that bears their names [20,22]. The fractures were categorized in three types based on articular displacement and the degree of comminution: Type I, intraarticular fracture with a nondisplaced articular surface; Type II, a fracture-dislocation with an incongruous joint; and Type III, a fracture with comminution of the distal tibia articular surface (Figs. 1, 2, 3) [22]. The original intended purpose of this classification system was primarily descriptive. Designing a simple system of describing the fracture pattern is critical in preoperative planning in the four-stage operative principle they had proposed. The severity of injury and its association with pain and functional outcomes were merely inferred. In early published reports, Rüedi and Allgöwer did not directly compare pain and
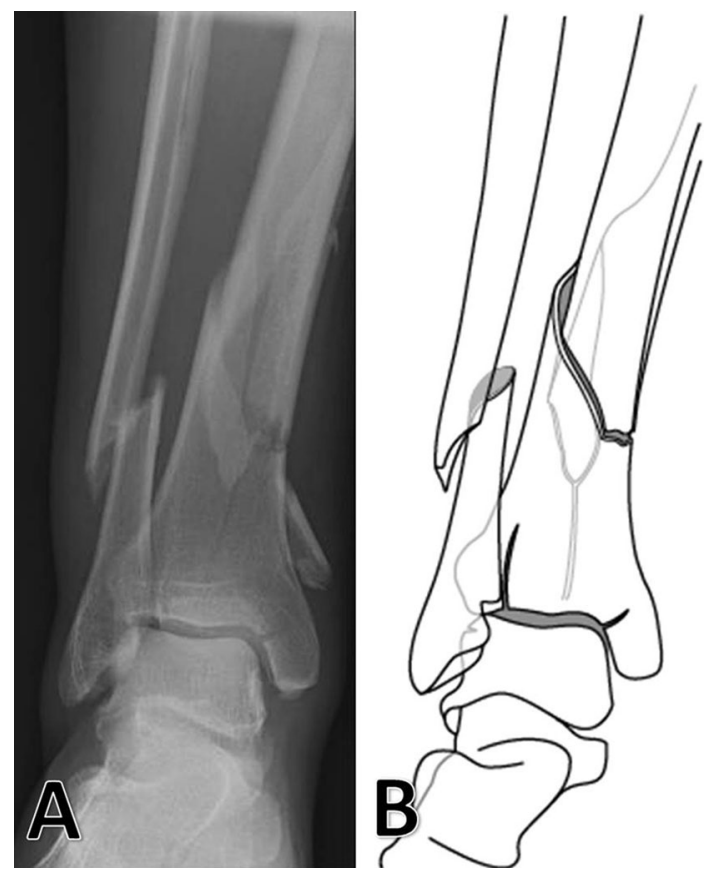

Fig. 1A-B A Type I Rüedi-Allgöwer tibial pilon fracture is shown in this (A) radiograph and in (B) the drawing. This is a simple fracture of the distal tibia with minimal articular displacement. tibiotalar joint motion among the three types of fracture patterns.

With time, the role of the classification system has expanded beyond comparison and descriptive purposes to determine prognosis and predict outcomes $[13,18,26]$.

\section{Description of the Rüedi-Allgöwer Classification}

Type I Rüedi-Allgöwer fractures (Fig. 1) are defined as "cleavage fractures" of the distal tibia without displacement [22]. Although the original classification did not specify the amount of displacement, a subsequent study considered displacement as greater than $2 \mathrm{~mm}$ of articular incongruity or greater than $10^{\circ}$ malreduction in any plane [30]. This type comprises the lowest percentage of pilon fractures, representing approximately $5 \%$ to $33 \%$ of fractures across numerous studies $[2,4,13,22,24,26,28,30]$. Type I fractures usually result from rotational injury with limited axial load [30]. Of the three types described under this system, only Type I fractures are associated with a high rate of satisfactory reduction using closed methods compared with Types II and III, largely owing to the lack of incongruity of the articular surface [18].

Type II fractures (Fig. 2) are characterized by moderate fracture-dislocation of the articular surface without comminution [22]. This is the second most common fracture

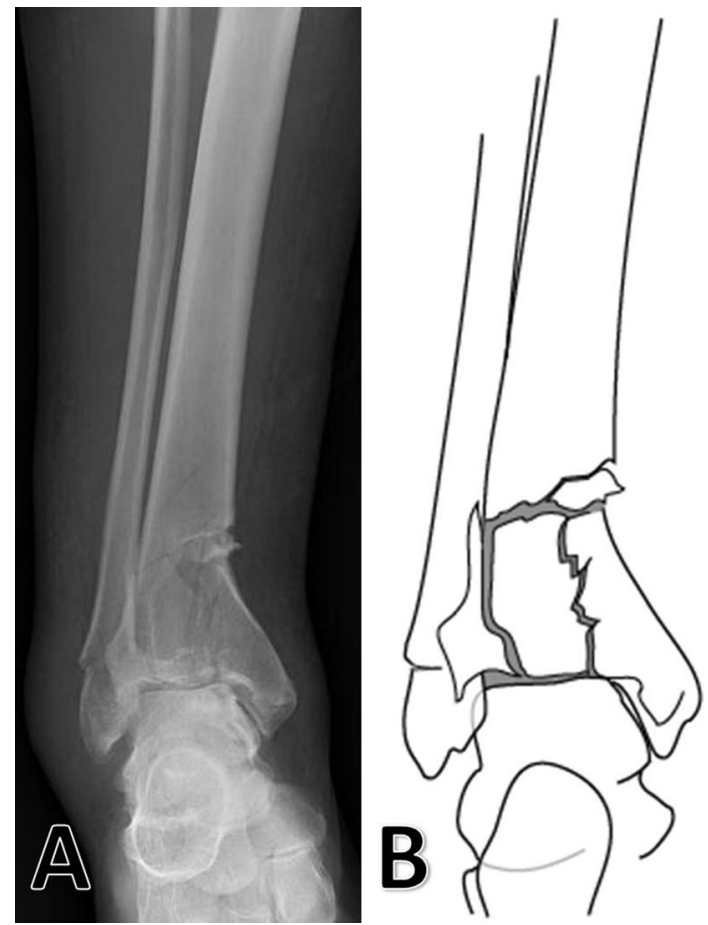

Fig. 2A-B A Type II Rüedi-Allgöwer fracture is shown in this (A) radiograph and in (B) the drawing. This is a moderate fracturedislocation of the distal tibial articular surface without comminution. 


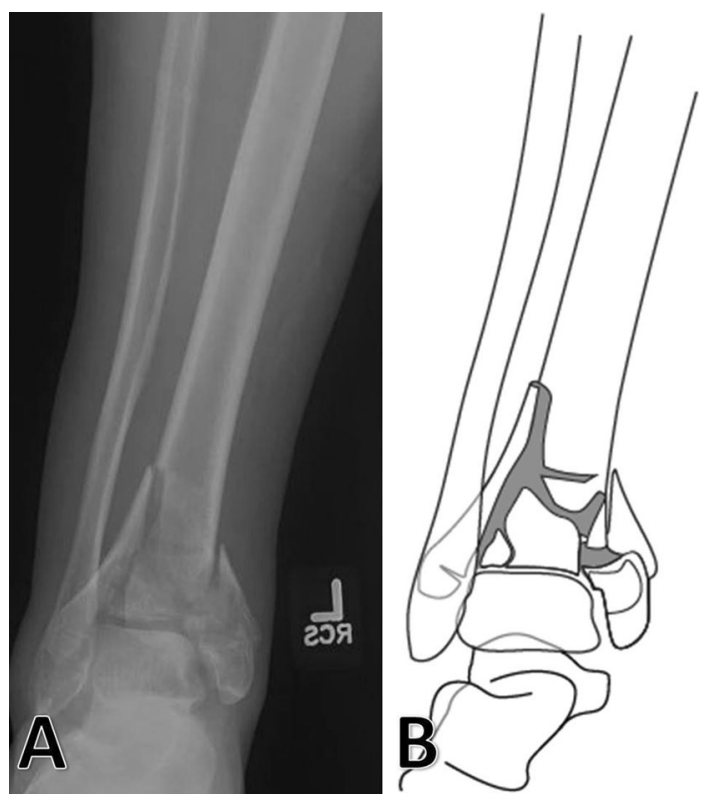

Fig. 3A-B A Type III Rüedi-Allgöwer fracture is shown in this (A) radiograph and in (B) the drawing. This fracture involves substantial comminution of the distal tibial articular surface with impaction of the metaphysis.

pattern observed under this classification system, comprising approximately $21 \%$ to $45 \%$ of pilon fractures $[2,4,13,22,24,26,28,30]$. Displacement of the articular surface in Type II fractures, resulting from a greater axial load mechanism compared with Type I fractures, generally calls for operative fixation to achieve joint congruency. Chen et al. [4] graded fewer excellent anatomic reductions during the immediate postoperative period for Type II fractures compared with Type I (36\% versus 62\%, respectively).

Type III fractures (Fig. 3), characterized by a high degree of comminution of the articular surface with impaction of the metaphysis, present the most challenging fractures to treat owing to the difficulty in achieving articular reduction and managing the soft tissue trauma [22]. This fracture pattern is the most commonly reported, representing approximately $25 \%$ to $71 \%$ of all pilon fractures in published studies $[2-4,13,22,24,26,28,30]$. The presence of comminution is indicative of an increased amount of axial load energy compared with Types I and II.

\section{Validation}

A good fracture classification system should provide a suitable description of the fractures to facilitate communication between surgeons, show adequate interobserver agreement, guide surgical decision-making, and offer a prognosis for pertinent outcomes in different groups of patients $[8,9,19]$. Several classification systems have been suggested for tibial pilon fractures; however, the RüediAllgöwer classification and the Arbeitsgemeinschaft für Osteosynthesefragen/Orthopaedic Trauma Association (AO/OTA) classification of long-bone fractures remain the two most commonly used today. Both of these systems attempt to depict the pattern and severity of the fracture.

Dirschl and Adams [8] first assessed the reliability of the Rüedi-Allgöwer classification among orthopaedic attendings and residents using AP or mortise and lateral radiographs. Twenty-five pilon fractures were reviewed and classified with and without the use of a binary decision tree composed of three yes or no questions meant to aid the observer in diagnosing the fracture type. The mean kappa values for the classification system and the modified binary system showed moderate reliability $(0.43$ and 0.35 , respectively). Martin et al. [14] reported a similar kappa value (0.46) among six reviewers of variable experience when asked to classify 43 pilon fractures according to the Rüedi-Allgöwer system. In comparison, the interobserver agreement was greater for classifying fractures in the $\mathrm{AO} /$ OTA types $(\mathrm{kappa}=0.60)$. Other authors have reported reliability in ranking the severity of pilon fractures [7, 9]. When 69 orthopaedic surgeons were asked to rank the severity of a series of 10 pilon fractures on AP and lateral radiographs from 1 (least severe) to 10 (most severe), the intraclass correlation coefficient (ICC) was 0.62 [9]. In an earlier study, three orthopaedic trauma surgeons ranked 25 pilon fractures according to the severity of injury to the articular surface and severity of the overall fracture pattern, yielding ICC values of 0.94 and 0.89 , respectively [7]. These high ICC values may be related to the small number of observers, but nevertheless highlight that pilon fracture characteristics vary on a continuum and are difficult to categorize based on classification schemes such as the Rüedi-Allgöwer system.

Advances in radiographic imaging with the advent of $\mathrm{CT}$ and three-dimensional reconstruction have improved the ability to discern fracture patterns with greater clarity, far beyond the simplistic descriptive capabilities afforded by the Rüedi-Allgöwer classification, making it less relevant today. Several studies have shown that categorizing pilon fractures into Rüedi-Allgöwer types based on CT does not reliably improve interobserver agreement. Topliss et al. [28] characterized a series of 126 pilon fractures to assess reproducibility on CT scans obtained before definitive fixation. The mean kappa values among five reviewers were 0.46 for the AO/OTA classification and 0.45 for the Rüedi-Allgöwer classification, showing an interobserver agreement on par with previous studies that relied on plain radiographs $[8,14]$. They proposed a new classification system using coronal and sagittal $\mathrm{CT}$ assessment after initial external fixation to bring the fracture out to length. 
Based on fracture lines observed on CT, they grouped 10 types of pilon fractures into two families (sagittal and coronal), which they postulated can influence the choice of surgical approach and implant to optimize fixation. Under this classification, mean kappa values were 0.67 for the fracture family and 0.58 for the fracture type. In the aforementioned study by Martin et al. [14], CT scans were available for 14 of the 43 fractures. For these 14 cases, interobserver agreement improved negligibly from the use of plain radiographs only (kappa $=0.52$ ) to the addition of CT (kappa = 0.53). Ramappa et al. [19] found similar interobserver agreement on pilon fracture assessment on $\mathrm{CT}$ for the Rüedi-Allgöwer classification $(\mathrm{kappa}=0.51)$, AO/OTA type $(\mathrm{kappa}=0.57)$, and Topliss type (kappa $=0.44)$.

The lack of articular surface congruity in pilon fractures is predictive of outcomes. Quality of reduction, as graded on immediate postoperative radiographs, correlates with greater subjective pain and functional satisfaction, greater objective gait and motion outcomes, and lower rate of progressive osteoarthritis $[4,13,18,26,30]$. Types I and II fractures are comparable with respect to the rate of satisfactory clinical outcomes (87\% versus $79 \%$, respectively) [4], defined on a 100-point scale for assessing ankle pain and function [16]. No pain with activity equates to 50 points. In addition, walking distance, support/orthosis requirement, ability to run, toe-raise, hill-climb, and stairclimb, presence of a limp or swelling, and ankle ROM also are evaluated. Teeny and Wiss [26] defined clinical outcomes as excellent ( $>92$ points), good (87 to 92 points), fair (65 to 86 points), or poor ( $<65$ points). In their series, Type III fractures resulted in a $13 \%$ rate of good to excellent outcomes compared with $37 \%$ for Types I and II fractures, owing to the increased difficulty in achieving adequate reduction of the comminuted fragments and maintaining stability of the fixation [26]. In a more recent series, despite advances in surgical technique, excellent results based on the 100-point scale were found in $15 \%$ of Type III fractures compared with $51 \%$ and $32 \%$ for Types I and II, respectively [4].

Type III Rüedi-Allgöwer fractures historically have shown higher complication rates compared with Types I and II (70\% versus 30\%) [26]. The complications reported by Teeny and Wiss [26], which included skin dehiscence, deep infections, malunion, and nonunion, were more common in Type III fractures compared with Types I and II fractures. The rate of reoperation was $60 \%$ for Type III fractures compared with $30 \%$ for Types I and II fractures. Type III fractures have been independently associated with worse functional outcomes; however, increased difficulty in achieving reduction and stable fixation compounds this issue [13]. When ankle ROM and functional outcomes were compared between fracture types in the AO/OTA classification, no association was found to suggest its effectiveness in predicting outcomes $[6,13]$.

\section{Limitations}

As familiarity with a system increases, so too does interobserver agreement [19]. The greater than 40-year history of the Rüedi-Allgöwer system has made it one of the more familiar classifications in use. Systems with fewer branching divisions further reduce the amount of interobserver disagreement [19]. With only three fracture types, the Rüedi-Allgöwer classification is the simplest classification system available to characterize tibial pilon fractures; however, several limiting factors exist.

First, in the original series by Rüedi and Allgöwer, the majority of fractures were skiing injuries that resulted in low-energy rotational deformities of the distal tibia [21]. This mechanism of injury typically results in metaphyseal displacement, which is not directly addressed by this classification, leading to proposed modifications by Ovadia and Beals [18] and Kapukaya et al. [11]. Subsequent series conducted in large urban trauma centers focused on highenergy mechanisms of injury (motor vehicle accidents and falls from height) that resulted in direct axial compression and articular impaction fractures of the tibial plafond $[4,13,24-26,30]$. The first surgical principle proposed by Rüedi and Allgöwer advocates for restoration of the fibular length, yet their classification system neither addresses the presence of a fibular fracture nor distinguishes malleolar fractures, which occur via different mechanisms.

Another drawback of the Rüedi-Allgöwer classification system is its limited ability to distinguish the location of articular fracture lines in comparison to the AO/OTA classification. It is the "simple" nature of the Rüedi-Allgöwer system that serves as a blessing and a curse. By limiting the number of subtypes, the Rüedi-Allgöwer classification differentiates fractures based on severity but either omits or oversimplifies the true nature of the fracture. Examples include extraarticular (AO/OTA Type 43A) and partial articular fractures (AO/OTA Type 43-B) or fractures involving the metaphysis (AO/OTA Types 43-C1 and 43-C2) [10]. These differences in morphologic features of fractures may alter surgical planning; for example, partial articular fractures (AO/OTA Type 43-B) may require a medial buttress plate as advocated by the last of the four operative principles, but may not be necessary in all pilon fractures.

Along a similar line, Type III fractures show highly variable morphologic features but are considered a uniform group in this classification [11]. The amount and severity of comminution cannot be extrapolated under the Rüedi-Allgöwer classification [1]. Several studies have shown 
difficulty in identifying articular fragments on plain radiographs [1, 8, 19]; therefore, grasping the complete clinical picture remains challenging. Soft tissue trauma is another major factor that is associated with a greater risk of deep infections, reoperations, and nonunion $[11,13,15,26,27,30]$. High-energy axial load injuries usually are associated with severe soft tissue damage [28]. As understanding of this fracture has improved, treatment strategies also have evolved from the pioneering principles taught by Rüedi and Allgöwer. The high rates of wound dehiscence and deep infections historically in complex pilon fractures highlighted the importance of soft tissue handling $[12,26]$. Wound complications occurred at much lower rates in the Rüedi-Allgöwer series [21, 22] compared with Type III fractures reported by Teeny and Wiss [26] and Kellam and Waddell [12], indicating that the higherenergy of injury may correlate with greater soft tissue complications despite similar fracture types. This finding further limits this classification system as a prognostic tool. Staged treatment therefore is indicated for higher-energy complex pilon fractures to allow soft tissue swelling to abate. In a randomized study, Wyrsch et al. [30] reported lower rates of wound complications and infections with external fixation compared with open reduction and internal fixation. Using a staged protocol of definitive fixation approximately 2 weeks after initial external fixation, Sirkin et al. [25] reported a wound complication rate of $3 \%$ in closed fractures and $11 \%$ in open fractures.

The advent of CT scanning has further improved the ability to delineate complex pilon fracture patterns and further limited the usefulness of the radiograph-based Rüedi-Allgöwer classification for surgical planning. Tornetta and Gorup [29] assessed 22 pilon fractures using CT, which provided additional information and altered the surgical plan in $82 \%$ and $64 \%$ of fractures, respectively. Under the staged protocol, CT of the tibial plafond typically is performed after external fixation brings the fracture to length. The added information provided by $\mathrm{CT}$ and three-dimensional reconstruction can assist the treating surgeon to determine incisional approach, implant choice, and plate placement.

\section{Conclusions}

The Rüedi-Allgöwer classification, although not a perfect classification system, remains one of the most widely known classification systems for pilon fractures. Its clinical relevance in providing a thorough description of the fracture pattern is limited and pales in comparison to the more comprehensive AO/OTA classification. Given the complex nature of these fractures, it stands to reason that an allinclusive fracture classification system would be inherently complex. Because of its historical significance and familiarity, the Rüedi-Allgöwer classification may continue to serve a role in the initial treatment decision; however, the advent of CT evaluation and staged reconstruction of complex pilon fractures have limited the use of this classification.

Acknowledgments We thank Eileen Elsner (Department of Orthopaedic Surgery, Wake Forest Baptist Medical Center, WinstonSalem, NC, USA) for assistance with the illustrations used in this study.

\section{References}

1. Anderson DD, Mosqueda T, Thomas T, Hermanson EL, Brown TD, Marsh JL. Quantifying tibial plafond fracture severity: absorbed energy and fragment displacement agree with clinical rank ordering. J Orthop Res. 2008;26:1046-1052.

2. Anglen JO. Early outcome of hybrid external fixation for fracture of the distal tibia. J Orthop Trauma. 1999;13:92-97.

3. Ballal A, Rai HR, Shetty SM, Mathias LJ, Shetty V, Shetty A. A prospective study on functional outcome of internal fixation of tibial pilon fractures with locking plate using minimally invasive plate osteosynthesis technique. J Clin Diagn Res. 2016;10:RC0104.

4. Chen $\mathrm{SH}, \mathrm{Wu} \mathrm{PH}$, Lee YS. Long-term results of pilon fractures. Arch Orthop Trauma Surg. 2007;127:55-60.

5. Cole PA, Mehrle RK, Bhandari M, Zlowodzki M. The pilon map: fracture lines and comminution zones in OTA/AO type 43C3 pilon fractures. J Orthop Trauma. 2013;27:e152-156.

6. Cutillas-Ybarra MB, Lizaur-Utrilla A, Lopez-Prats FA. Prognostic factors of health-related quality of life in patients after tibial plafond fracture: a pilot study. Injury. 2015;46:2253-2257.

7. DeCoster TA, Willis MC, Marsh JL, Williams TM, Nepola JV, Dirschl DR, Hurwitz SR. Rank order analysis of tibial plafond fractures: does injury or reduction predict outcome? Foot Ankle Int. 1999;20:44-49.

8. Dirschl DR, Adams GL. A critical assessment of factors influencing reliability in the classification of fractures, using fractures of the tibial plafond as a model. J Orthop Trauma. 1997;11:471476.

9. Dirschl DR, Ferry ST. Reliability of classification of fractures of the tibial plafond according to a rank-order method. J Trauma. 2006;61:1463-1466.

10. Jacob N, Amin A, Giotakis N, Narayan B, Nayagam S, Trompeter AJ. Management of high-energy tibial pilon fractures. Strategies Trauma Limb Reconstr. 2015;10:137-147.

11. Kapukaya A, Subasi M, Arslan H, Tuzuner T. Non-reducible, open tibial plafond fractures treated with a circular external fixator (is the current classification sufficient for identifying fractures in this area?). Injury. 2005;36:1480-1487.

12. Kellam JF, Waddell JP. Fractures of the distal tibial metaphysis with intra-articular extension: the distal tibial explosion fracture. J Trauma. 1979;19:593-601.

13. Korkmaz A, Ciftdemir M, Ozcan M, Copuroglu C, Saridogan K. The analysis of the variables, affecting outcome in surgically treated tibia pilon fractured patients. Injury. 2013;44:1270-1274.

14. Martin JS, Marsh JL, Bonar SK, DeCoster TA, Found EM, Brandser EA. Assessment of the AO/ASIF fracture classification for the distal tibia. J Orthop Trauma. 1997;11:477-483.

15. Mauffrey C, Vasario G, Battiston B, Lewis C, Beazley J, Seligson D. Tibial pilon fractures: a review of incidence, diagnosis, 
treatment, and complications. Acta Orthop Belg. 2011;77:432440.

16. Mazur JM, Schwartz E, Simon SR. Ankle arthrodesis: long-term follow-up with gait analysis. J Bone Joint Surg Am. 1979;61:964975.

17. Michelson J, Moskovitz P, Labropoulos P. The nomenclature for intra-articular vertical impact fractures of the tibial plafond: pilon versus pylon. Foot Ankle Int. 2004;25:149-150.

18. Ovadia DN, Beals RK. Fractures of the tibial plafond. $J$ Bone Joint Surg Am. 1986;68:543-551.

19. Ramappa M, Bajwa A, Singh A, Mackenney P, Hui A, Port A. Interobserver and intraobserver variations in tibial pilon fracture classification systems. Foot (Edinb). 2010;20:61-63.

20. Rüedi TP. Fractures of the lower end of the tibia into the ankle joint: results 9 years after open reduction and internal fixation. Injury. 1973;5:130-134.

21. Rüedi TP, Allgöwer M. Fractures of the lower end of the tibia into the ankle-joint. Injury. 1969;1:92-99.

22. Rüedi TP, Allgöwer M. The operative treatment of intra-articular fractures of the lower end of the tibia. Clin Orthop Relat Res. 1979;138:105-110.

23. Rüedi TP, Matter P, Allgöwer M. Intra-articular fractures of the distal tibial end [in German]. Helv Chir Acta. 1968;35:556-582.
24. Schulz AP, Fuchs S, Simon L, Seide K, Paech A, Queitsch C. Severe fracture of the tibial pilon: results with a multidirectional self-locking osteosynthesis plate utilizing a two-stage procedure. Eur J Trauma Emerg Surg. 2008;34:391-396.

25. Sirkin M, Sanders R, DiPasquale T, Herscovici D Jr. A staged protocol for soft tissue management in the treatment of complex pilon fractures. J Orthop Trauma. 1999;13:78-84.

26. Teeny SM, Wiss DA. Open reduction and internal fixation of tibial plafond fractures: variables contributing to poor results and complications. Clin Orthop Relat Res. 1993;292:108-117.

27. Thordarson DB. Complications after treatment of tibial pilon fractures: prevention and management strategies. J Am Acad Orthop Surg. 2000;8:253-265.

28. Topliss CJ, Jackson M, Atkins RM. Anatomy of pilon fractures of the distal tibia. J Bone Joint Surg Br. 2005;87:692-697.

29. Tornetta P 3rd, Gorup J. Axial computed tomography of pilon fractures. Clin Orthop Relat Res. 1996;323:273-276.

30. Wyrsch B, McFerran MA, McAndrew M, Limbird TJ, Harper MC, Johnson KD, Schwartz HS. Operative treatment of fractures of the tibial plafond: a randomized, prospective study. $J$ Bone Joint Surg Am. 1996;78:1646-1657. 